\title{
Activation of Constitutive Androstane Receptor Prevents Cholesterol Gallstone Formation
}

Shihai Cheng, ${ }^{* \dagger}$ Min Zou, ${ }^{\ddagger}$ Qinhui Liu, ${ }^{\dagger}$ Jiangying Kuang, ${ }^{\dagger \dagger}$ Jing Shen, ${ }^{\dagger \ddagger}$ Shiyun Pu, ${ }^{\dagger \dagger}$ Lei Chen, ${ }^{\dagger \ddagger}$ Hong Li, ${ }^{\dagger \dagger}$ Tong Wu, ${ }^{\dagger \ddagger}$ Rui Li, $^{\dagger \dagger}$ Yanping $\mathrm{Li}_{1}^{\dagger \ddagger}$ Wei Jiang, ${ }^{\S}$ Zhiyong Zhang, and Jinhan $\mathrm{He}^{\dagger \ddagger}$

From the Department of Clinical Pharmacy and Pharmacy Administration, * West China School of Pharmacy, Sichuan University, Chengdu; and the Laboratory of Clinical Pharmacy and Adverse Drug Reaction, ${ }^{\dagger}$ the Department of Pharmacy, ${ }^{\ddagger}$ and the Molecular Medicine Research Center, ${ }^{\S}$ State Key Laboratory of Biotherapy, West China Hospital of Sichuan University, Chengdu, China

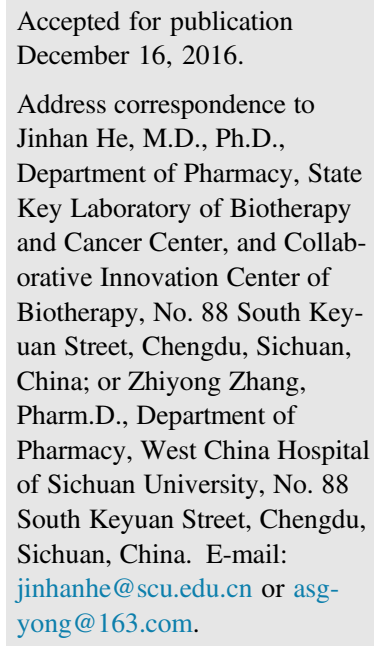

Cholesterol gallstone disease (CGD) is one of the most common gastrointestinal diseases. Lithogenic hepatic bile secretion precedes the formation of cholesterol gallstones. Constitutive androstane receptor (CAR), a member of nuclear family, plays an important role in cholesterol and bile acid metabolism. To examine whether activation of CAR can prevent cholesterol gallstone formation, we treated C57BL6/J mice maintained on a lithogenic diet with CAR agonist 1,4-bis-[2-(3, 5-dichlorpyridyloxy)] benzene and performed bile duct cannulation to study the dynamics of biliary lipids. We report that activation of CAR decreases the biliary cholesterol concentration and prevents CGD formation. The lower biliary cholesterol level was largely attributed to suppressed Abcg5 and Abcg8 expression in CAR-activated mice. CAR activation also promoted cholesterol conversion into bile acids by increasing the expression of Cyp7a1, a rate-limiting enzyme in bile acid biosynthesis. Activation of CAR enhanced bile acid re-absorption via increasing the expression of bile acid transporters Asbt and Ost $\beta$ in the ileum. The hepatic steatosis was also improved in the liver of CAR-activated mice. Furthermore, activation of CAR protected the mice against the liver $X$ receptor $\alpha$-sensitized CGD through suppressing the expression of Abcg5/8. Collectively, CAR plays an important role in maintaining the homeostasis of cholesterol, bile acids, and triglycerides levels, and it might be a promising therapeutic target for preventing or treating CGD. (Am J Pathol 2017, 187: 808-818; http://dx.doi.org/10.1016/j.ajpath.2016.12.013)
Cholesterol gallstone disease (CGD) is one of the most common gastroenterologic disorders. The incidence rate is increasing because of improved standards of living, a chronic high-cholesterol diet, and overnutrition. The precipitation of excess cholesterol in the bile as solid crystals is a prerequisite for cholesterol gallstone formation. In the normal physiological state, cholesterol can be solubilized in mixed micelles composed of phospholipids and bile salts, all in dynamic equilibrium. However, under certain pathophysiological conditions, especially the supersaturated bile of cholesterol, the relative excess cholesterol will precipitate as solid crystals, then aggregate, fuse, and eventually form gallstones that cause disease. ${ }^{1}$

The main components of bile are cholesterol, phospholipids, and bile acids (BAs), which are controlled by an elaborate network of transporters. Cholesterol exits the body principally by efficient secretion into bile, mediated by the
ATP-binding cassette (Abc) transporters Abcg5 and Abcg8. ${ }^{2,3}$ The canalicular efflux of phospholipids depends on Abcb4, also known as multidrug resistance protein 2 (Mdr2). ${ }^{4}$ Abca1 is a basolateral transporter that effluxes both cholesterol and phospholipids. The primary BAs are synthesized from cholesterol in the liver controlled by multiple cytochrome P450 (Cyp) enzymes via the classical pathway (mainly mediated by Cyp7a1 and Cyp8b1) or alternative pathways (mainly controlled by Cyp7b1 and

\footnotetext{
Supported by the National Natural Science Foundation of China grants 81270926 and 81471068 (J.H.), Distinguished Young Scientists of Sichuan Province grant 2014JQ0034 (J.H.), and Young Scientist Fellowship of Sichuan University grant 2013SCU04A17 (J.H.).

S.C. and M.Z. contributed equally to this work.

Disclosures: None declared.
} 
Cyp27a1), which accounts for approximately $75 \%$ and $25 \%$ of the total primary BAs, respectively. ${ }^{5}$

Cyp7a1 is the rate-limited enzyme and can be suppressed by the negative feedback regulation of BA synthesis. Cyp7a1 is down-regulated by small heterodimerizing partner (Shp) in the liver and fibroblast growth factor 15 (Fgf15) in the small intestine. ${ }^{6-8}$ Fgf15, derived from the small intestine, circulates in the blood to the liver, binds to the Fgf receptor 4 (Fgfr4)/ $\beta$-Klotho receptor on the surface of hepatocytes to repress the expression of Cyp7a1. ${ }^{7}$ When emptied from the gallbladder to the intestine, $>90 \%$ of BAs can be reabsorbed by the apical sodium-dependent BA transporter (Asbt) and basolateral organic solute transporter $\alpha / \beta(O s t \alpha / \beta)$ in the ileum and then circulates back to the liver through the hepatic uptake transporters sodium $\left(\mathrm{Na}^{+}\right)$taurocholate cotransport protein (Ntcp) and the organic anion transporting polypeptides (Oatps). ${ }^{9}$ Both newly synthesized and reabsorbed BAs are secreted into the bile by the BA export pump (Bsep) and multidrug-resistance protein 2 (Mrp2). Mrp3 and Mrp4 play a role in the sinusoid export of conjugated bile salts and promote their renal secretion. ${ }^{10}$

Constitutive androstane receptor (CAR), primarily considered a xenobiotic sensor, is predominantly expressed in liver and small intestine. ${ }^{11}$ It mediates specific xenobiotic induction of drug metabolism and plays an important role in stimulating BAs and bilirubin detoxification and elimination. ${ }^{12}$ CAR activation can increase the elimination of cholesterol by its conversion into BAs and subsequent fecal excretion, with positive consequences on reverse cholesterol transport and whole-body cholesterol homeostasis. ${ }^{13}$ Despite the role of CAR in the elimination of BAs in liver, a recent study showed that CAR activation still maintains the biliary excretion of BAs. ${ }^{14}$ In addition, CAR plays an important role in preventing atherosclerosis. ${ }^{13,15}$ However, the effect of CAR activation on the pathogenesis of CGD remains unclear.

In this study, we examined activation of the nuclear receptor CAR in preventing cholesterol gallstone formation in mice. We treated C57BL6/J mice with the CAR agonist 1,4bis-[2-(3,5-dichlorpyridyloxy)] benzene (TCPOBOP) and simultaneously fed them with a lithogenic diet (LGD). Activation of CAR decreased the biliary cholesterol concentration and prevented CGD formation. The lower biliary cholesterol level was largely attributed to suppressed Abcg5 and Abcg8 expression in CAR-activated mice. CAR activation also promoted cholesterol conversion to BAs by increasing Cyp7a1 expression. Modulation of CAR activity might be a therapeutic target for CGD formation.

\section{Materials and Methods}

\section{Animals, LGD, and Drug Treatment}

All animal care and experimental procedures complied with the guidelines of the Animal Care and Utilization
Committee of the institute and were approved by this committee. Eight-week-old C57BL6/J male mice were purchased from Sichuan University Laboratory Animal center (Chengdu, China), housed with a 12:12-hour light/ dark cycle, and permitted ad libitum consumption of water and a standard mouse diet. When necessary, mice received an intraperitoneal injection of vehicle or the CAR ligand TCPOBOP (sc-203291; Santa Cruz Biotechnology, Santa Cruz, CA) $1.0 \mathrm{mg} / \mathrm{kg}$ body weight, once per week. The vehicle- or TCPOBOP-treated mice were fed a chow diet (CD) or LGD (Harlan Teklad TD.88051) containing approximately $15.8 \%$ fat, $1.25 \%$ cholesterol, $0.5 \%$ sodium cholate, and $7.5 \%$ casein for 2 weeks. The TCPOBOP was injected 1 day before the start of LGD feeding, and the drug treatment was continued until the end of the experiments. After 2 weeks, mice were sacrificed after being deprived of food overnight, and gallbladders were removed by ligation of the cystic duct. Gallbladder bile and contents were gently squeezed into tubes after cholecystectomy and immediately examined under a polarized microscope for cholesterol crystals and gallstones. The remaining bile was stored at $-20^{\circ} \mathrm{C}$ for biochemical analysis. Livers, gallbladders, and small intestines were frozen in liquid nitrogen and stored at $-80^{\circ} \mathrm{C}$. To demonstrate whether CAR activation can prevent liver X receptor (LXR) $\alpha$-sensitized gallstone formation, mice were treated with intraperitoneal injection of TCPOBOP (once a week) and daily gavage of $30 \mathrm{mg} / \mathrm{kg}$ GW3965, and then fed with LGD for 2 weeks.

\section{Bile Duct Cannulation and Collection of Hepatic Bile}

Bile duct cannulation was performed as described. ${ }^{16}$ Briefly, mice were deprived of food overnight and were anesthetized with isoflurane. The lower end of the common bile duct of mice was ligated, and the common bile duct was cannulated with a PE-10 polyethylene catheter below the entrance of the cystic duct. The cystic duct was then doubly ligated, and a cholecystectomy was performed. Hepatic bile was collected in $0.2-\mathrm{mL}$ tubes by gravity for 1 hour.

Histologic Evaluation and Biochemical Analysis of Bile, Hepatic Bile, Serum, Urine, and Feces

Livers and gallbladders were fixed in $10 \%$ formalin, embedded in paraffin, sectioned at $4 \mu \mathrm{m}$, and stained with hematoxylin and eosin. Frozen sections $(6 \mu \mathrm{m})$ were used for Oil Red O staining. Images were captured by a microscope (Nikon, Tokyo, Japan). Biliary, hepatic bile, and serum BA, cholesterol, and triglyceride levels were measured with assay kits from Biosino (Beijing, China). Phospholipids were measured by using a kit from Wako (296-63801; Osaka, Japan). The cholesterol saturation index (CSI) of bile and hepatic bile was calculated according to Carey's critical tables. ${ }^{17}$ To determine urinary and fecal BA excretion, mice were individually housed, and urine and feces were collected. Fecal BAs were extracted 

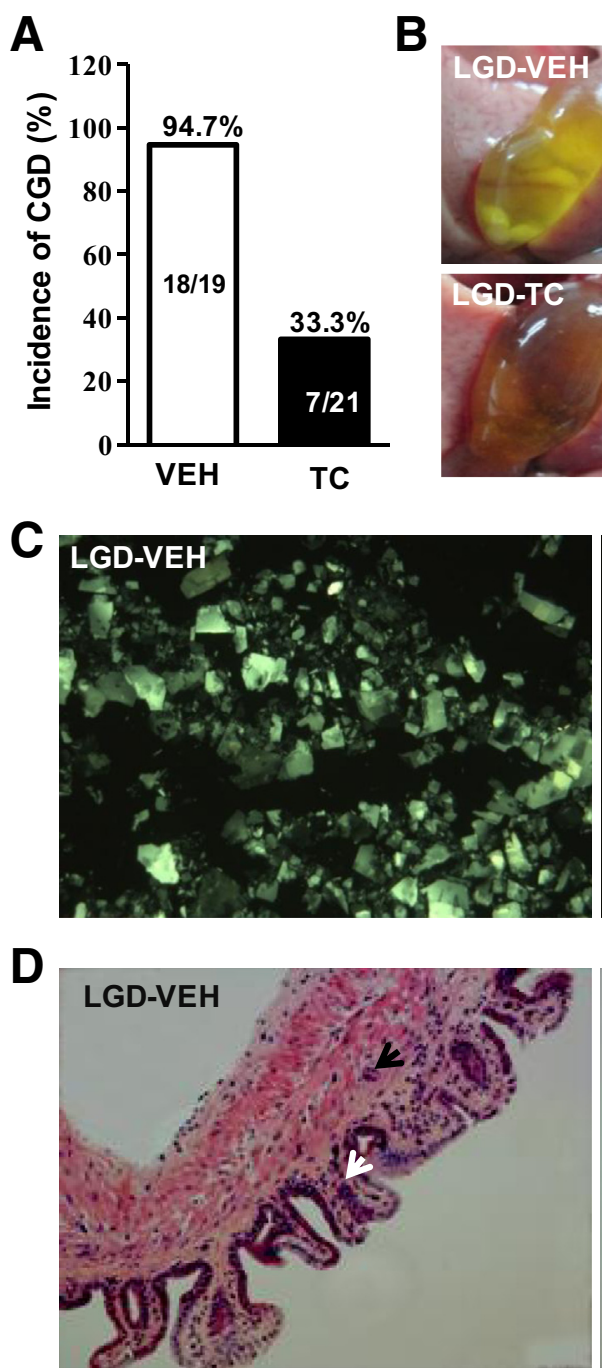
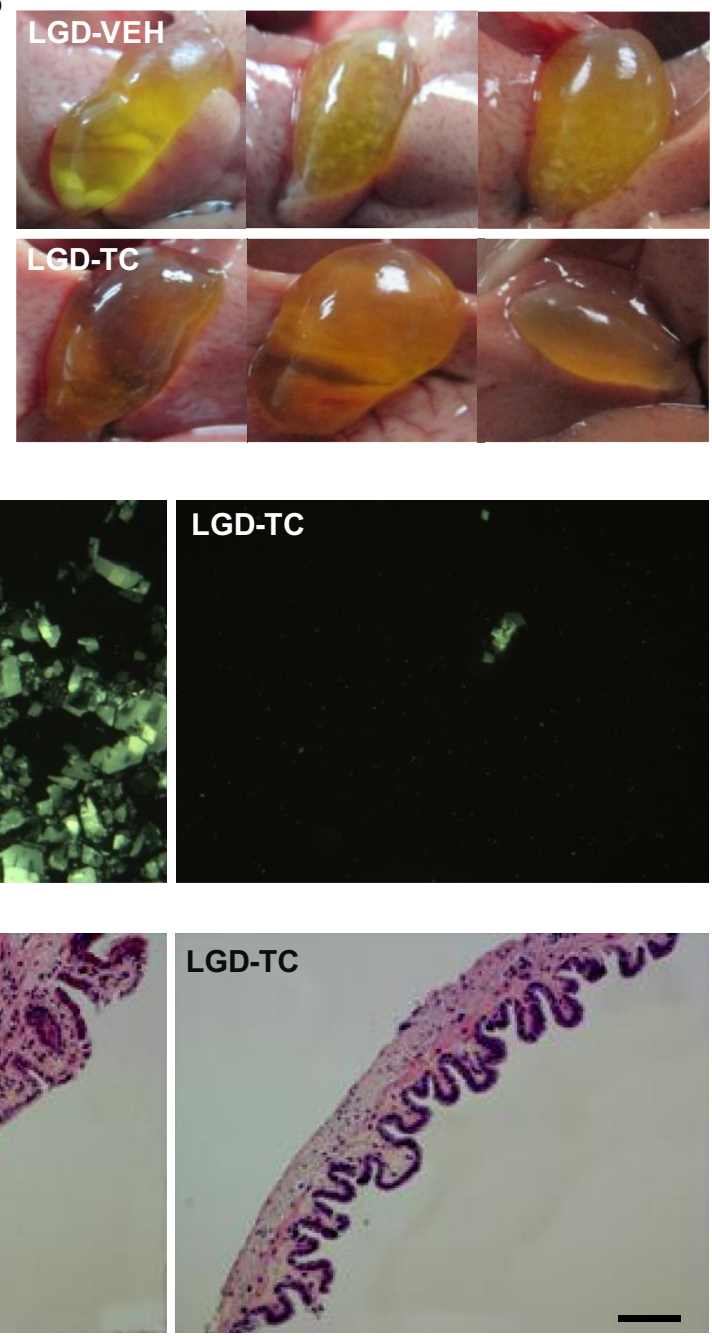

Figure 1 Activation of constitutive androstane receptor (CAR) prevents cholesterol gallstone disease (CGD) in C57BL/6J mice. A: Proportion of crystals in male $\mathrm{C} 57 \mathrm{BL} / 6 \mathrm{~J}$ mice treated with vehicle (VEH) and TC $(1.0 \mathrm{mg} / \mathrm{kg}$ body weight, once per week). All mice were fed a LGD for 2 weeks. The number of mice is indicated. B: Gross appearance of representative gallbladders. C: Polarizing light microscopy of cholesterol crystals. D: Histologic examination of gallbladder by H\&E staining. The white arrow indicates the thickness of the gallbladder wall. The black arrow indicates submucosal vasodilatation. $n=5$ per group. Scale bar $=50$ $\mu \mathrm{m}$ (D). Original magnification, $\times 100$ (C). H\&E, hematoxylin and eosin; LGD, lithogenic diet; TC, TCРОВОР or 1,4-bis-[2-(3,5-dichlorpyridyloxy)] benzene; VEH, vehicle. as described. ${ }^{18}$ Briefly, $0.5 \mathrm{~g}$ of dried feces was extracted in $10 \mathrm{~mL}$ of $75 \%$ ethanol at about $50^{\circ} \mathrm{C}$ for 2 hours, followed by centrifugation at $1500 \times g$ for 10 minutes. BAs were then measured enzymatically in the resulting supernatant fluid. The daily feces output (in $\mathrm{g} / \mathrm{d}$ per $100 \mathrm{~g}$ body weight) and fecal BA content (in $\mu \mathrm{mol} / \mathrm{d}$ per $100 \mathrm{~g}$ body weight) were used to calculate the rate of BA excretion. The total BA pool size was determined as BA content of the small intestine, the gallbladder, the liver, and their contents. ${ }^{18}$ After the mice were weighed, anesthetized, and exsanguinated, the fresh organs were collected, minced together, and extracted with $75 \%$ ethanol at about $50^{\circ} \mathrm{C}$ for 2 hours. The extract was centrifuged, BAs were determined enzymatically, and the pool size was expressed as micromoles of BAs per $100 \mathrm{~g}$ of body weight.

\section{Hepatic Lipid Analysis}

To analyze hepatic triglycerides and cholesterol levels, 150 $\mathrm{mg}$ of liver tissue was lysated into $1 \mathrm{~mL}$ of phosphatebuffered saline by homogenization. Total lipids were extracted by chloroform/methanol $(2: 1, \mathrm{v} / \mathrm{v})^{19}$ and dissolved in $1 \%$ Triton-X100 in ethanol. Triglyceride and cholesterol levels were measured by commercial assay kits from Biosino.

\section{Hepatic Total BA Analysis}

Hepatic total BAs were extracted as described. ${ }^{20}$ Briefly, liver tissues were homogenized in $75 \%$ ethanol and incubated at $50^{\circ} \mathrm{C}$ for 2 hours. The extracted supernatant fluids were assayed by using commercial assay kits from Biosino.

\section{Western Blot Analysis}

Liver and ileum were lyzed in lysis buffer and subjected to Western blot analysis with the following primary antibodies: anti-Abcg5 (dilution 1:1000; sc-25796), anti-Abcg8 (dilution 1:1000; sc-30111), and anti-Fgf15 (dilution 1:1000; sc-27177) from Santa Cruz Biotechnology; anti-Cyp7a1 (dilution 1:1000; ab-65596) from Abcam (Cambridge, MA); anti- $\beta$-actin (dilution 1:4000, A-4700) from Sigma-Aldrich (St. Louis, MO). Blots were visualized by LI-COR (Lincoln, 
A
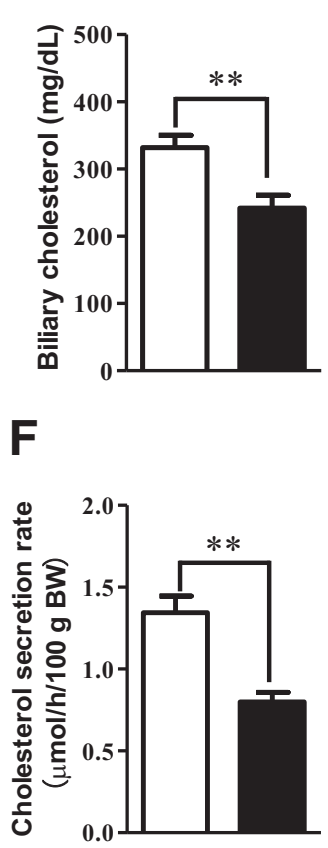

B
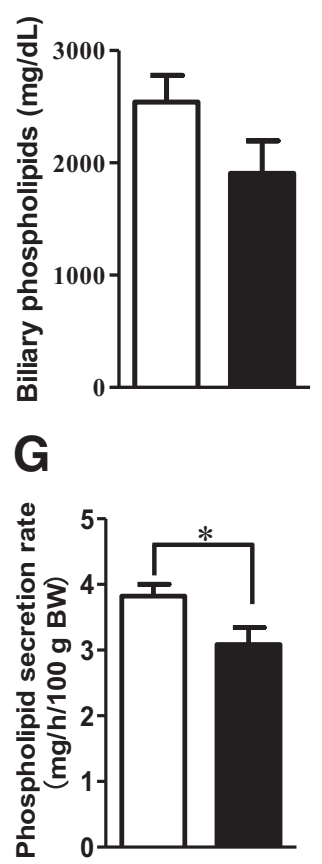

C

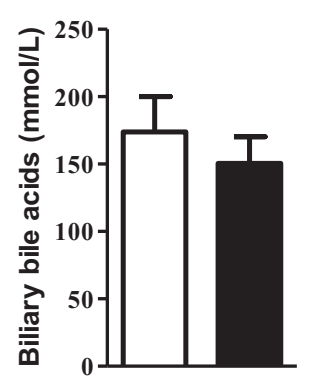

H

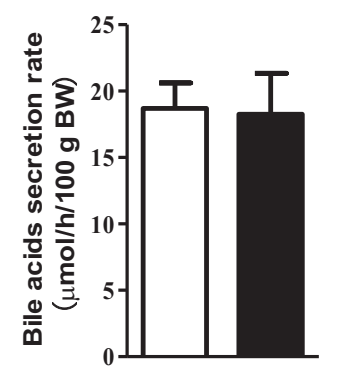

D
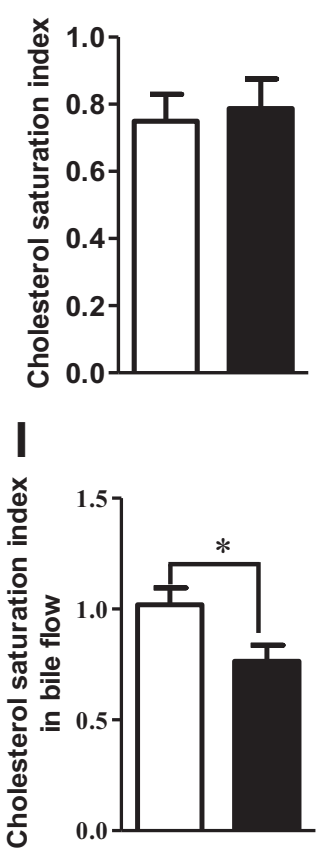

E

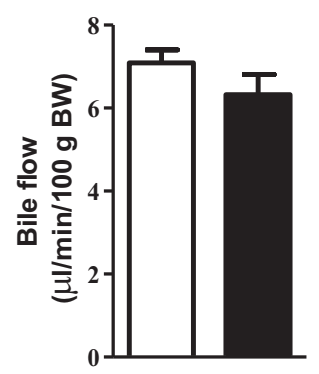

$\mathbf{J}$

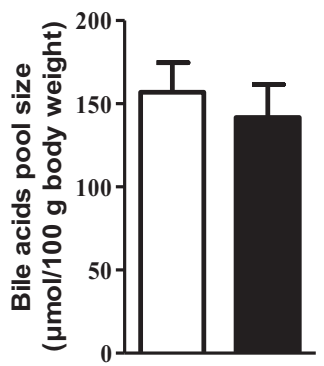

\section{$\square$ LGD-VEH $\square$ LGD-TC}

Figure 2 Activation of CAR alters the biochemical composition of the gallbladder and hepatic bile for antilithogenesis effects. A-D: Biliary concentrations of cholesterol (A), phospholipids (B), bile acids (C), and cholesterol saturation index (CSI) (D) of gallbladder bile. Male C57BL/6J mice treated with VEH and TC $(1.0 \mathrm{mg} / \mathrm{kg}$ body weight, once per week). All mice were fed an LGD for 2 weeks. E-I: Hepatic bile flow rate (E) and secretary rate of cholesterol (F), phospholipids (G), bile acids (H), and CSI (I). Bile was collected from the common bile duct of 10-week-old mice. J: Bile acid pool size. Data are expressed as means \pm SEM. $n=5$ per group (A-D); $n=7$ for each group $(\mathbf{E}-\mathbf{I}) ; n=5$ for each group (J). ${ }^{*} P<0.05, * * P<0.01$. BW, body weight; CAR, constitutive androstane receptor; LGD, lithogenic diet; TC, TCPOBOP or 1,4-bis-[2-(3,5-dichlorpyridyloxy)] benzene; VEH, vehicle.

NE) Odyssey System. Quantitative determination of band intensity was conducted by Image Studio analysis software version 4.0 (LI-COR).

\section{Quantitative Real-Time RT-PCR}

Total hepatic and ileal RNA was extracted with TRIzol reagent and reverse-transcribed into complementary DNA (RR037A; TaKaRa, Kyoto, Japan). Quantitative real-time PCR (no. 1708882AP; Bio-Rad, Hercules, CA) involved the SYBR Greenbased assay with the CFX96 Real-Time system.

\section{Statistical Analysis}

Data are expressed as means \pm SEM. One-way analysis of variance (Tukey's test) involved the use of GraphPad Prism version 5.0 (GraphPad Inc., San Diego, CA). $P<0.05$ was considered statistically significant.

\section{Results}

\section{Activation of CAR Prevents CGD in C57BL/6J Mice}

To determine the effect of CAR on lithogenesis, C57BL/6J mice were fed a CD or LGD for 2 weeks with or without the
CAR agonist TCPOBOP. With a $\mathrm{CD}$, vehicle- or TCPOBOP-treated mice showed no cholesterol crystals (Supplemental Figure S1A). However, with the LGD, the incidence of gallstones was $94.7 \%$ in vehicle-treated mice but decreased to $33.3 \%$ in TCPOBOP-treated mice (Figure 1A). The gallbladder in TCPOBOP-treated mice showed transparent bile, almost without any impurities compared with vehicle-treated mice that were filled with gallstones (Figure 1B). Polarizing light microscopy examination of bile revealed aggregated cholesterol crystals in vehicle-treated gallbladders, whereas TCPOBOP-treated mice were generally free of cholesterol precipitates (Figure 1C). Two-week LGD treatment caused inflammation in vehicle-treated mice, including thickening of gallbladder wall and submucosal vasodilatation. ТСРОВОР treatment ameliorated these signs of inflammation (Figure 1D).

Activation of CAR Alters the Biochemical Composition of the Gallbladder and Hepatic Bile for Antilithogenesis Effects

The protective effect of CAR activation on CGD prompted us to detect the biliary biochemical composition in the gallbladder. TCPOBOP-treated mice consuming a CD had 


\section{CD-TC}

LGD-VEH

LGD-TC

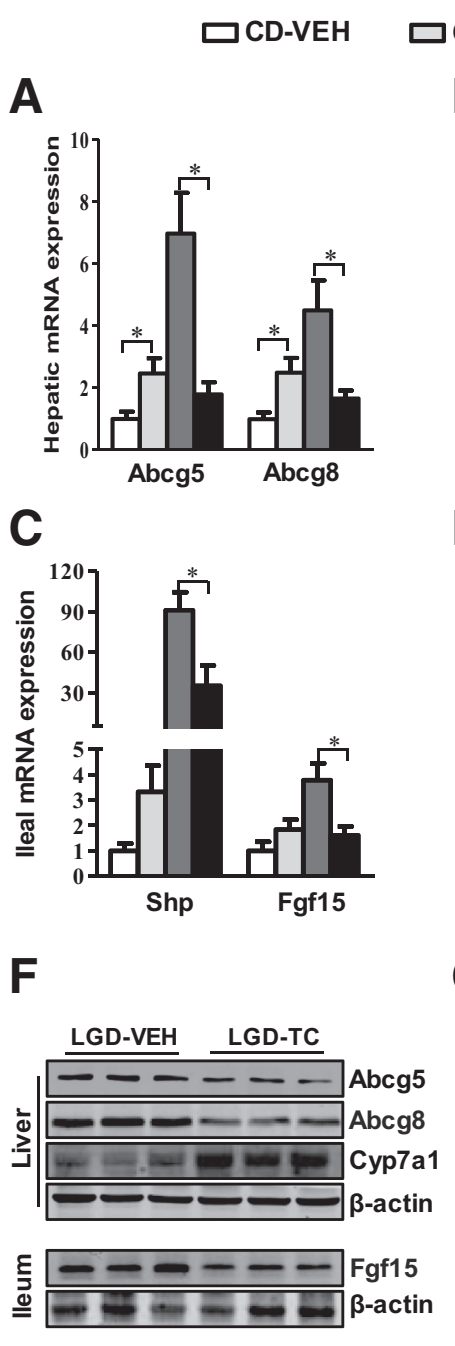

A

B
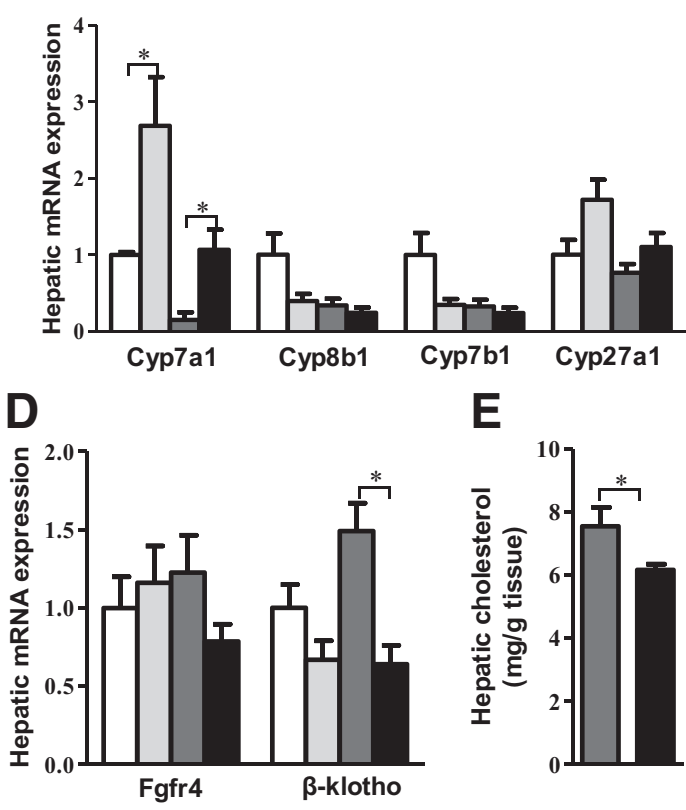

E

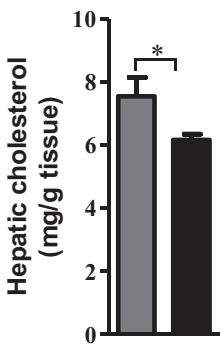

H

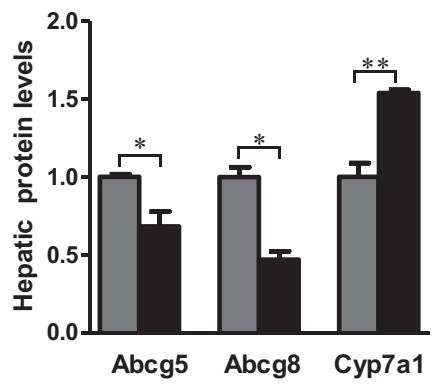

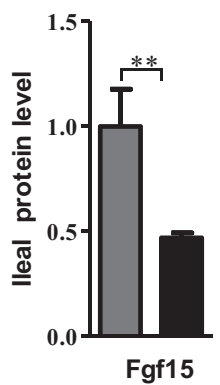

Figure 3 Activation of CAR decreases canalicular efflux of cholesterol and increases Cyp7a1 expression. A and B: Real-time PCR analysis of hepatic mRNA expression of cholesterol canalicular transporters (A) and bile acid synthesis enzyme genes (B). C: Ileal mRNA expression of Shp and Fgf15. D: Hepatic mRNA expression of Fgfr4 and $\beta$-klotho. E: Hepatic level of cholesterol. $\mathbf{F}$ : Expression of hepatic Abcg5/8, Cyp7a1, and ileal Fgf15 proteins were measured by Western blot analysis. $\mathbf{G}$ and $\mathbf{H}$ : Quantitative determination of band intensity in panel $\mathbf{F}$. Data are expressed as means \pm SEM. $n=5$ to 6 for each group. ${ }^{*} P<0.05,{ }^{*} P<0.01$. CAR, constitutive androstane receptor; $C D$, chow diet; LGD, lithogenic diet; TC, TCPOBOP or 1,4-bis[2-(3,5-dichlorpyridyloxy)] benzene; VEH, vehicle. similar biliary cholesterol, BAs, and phospholipid levels as vehicle-treated mice (Supplemental Figure S1, B-D). However, with 2 weeks of LGD feeding, TCPOBOP-treated mice showed significantly decreased biliary cholesterol concentration (Figure 2A) and slightly decreased phospholipid levels (Figure 2B). The biliary BA concentration was comparable in vehicle- and TCPOBOP-treated mice (Figure 2C). Despite its protective effect against lithogenesis, TCPOBOP treatment did not decrease biliary CSI in this phenotype (Figure 2D).

To further investigate the effect of CAR activation to prevent cholesterol gallstone formation, we performed bile duct cannulation to clarify the dynamics of bile flow and fresh biliary lipid outputs after a LGD. TCPOBOP did not change the bile flow but substantially the decreased biliary cholesterol secretion rate (Figure 2, E and F). The decreased cholesterol secretion rate was accompanied by a mild decrease in phospholipid secretion (Figure 2G) and constant BA output (Figure $2 \mathrm{H}$ ). As a result, TCPOBOP treatment decreased CSI in hepatic bile flow (Figure 2I). The decreased hepatic biliary CSI provided a pathophysiological explanation for the protective role of CAR activation in the lithogenic phenotype. Consistent with the similar secretion rate of BAs, total pool size of BAs in the mice of the two groups were equally comparable (Figure 2J).

\section{Activation of CAR Decreases Canalicular Efflux of Cholesterol and Increases Cyp7a1 Expression in Liver}

To gain insights into the molecular mechanisms by which CAR activation prevented cholesterol gallstone formation in C57BL6/J mice, first, we evaluated the expression levels of the genes involved in cholesterol, phospholipid, and BA transport. The mRNA expression of Cyp2b10, a target gene of CAR, was highly induced with both CD and LGD feeding after TCPOBOP treatment, indicating successful CAR activation (Supplemental Figure S2). In the liver, cholesterol is secreted by the canalicular transporters Abcg5 and Abcg8 or is converted into BAs via classic and alternative pathways that generate approximately $75 \%$ and $25 \%$ of the total primary BAs, respectively. ${ }^{21}$ Activation of CAR 

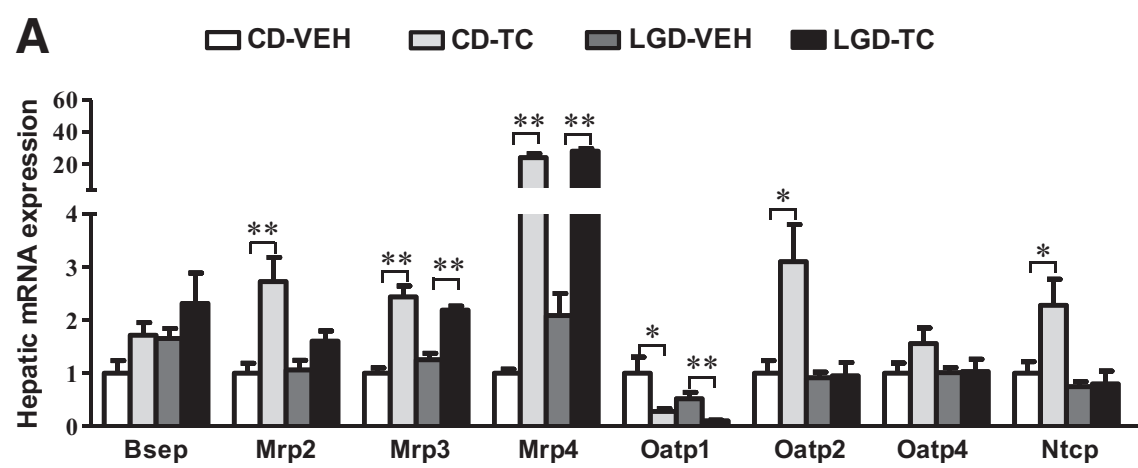

B

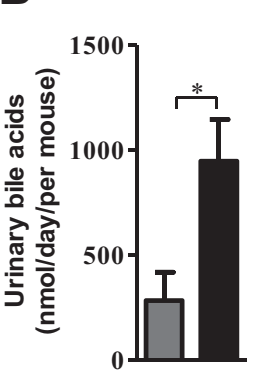

C

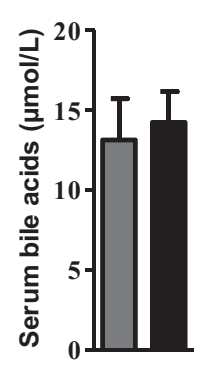

D

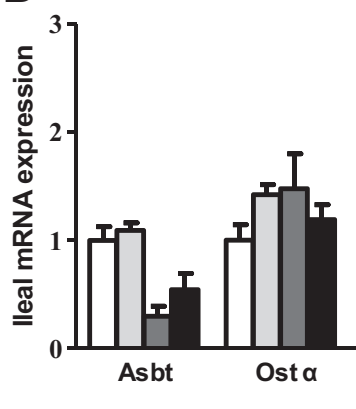

F

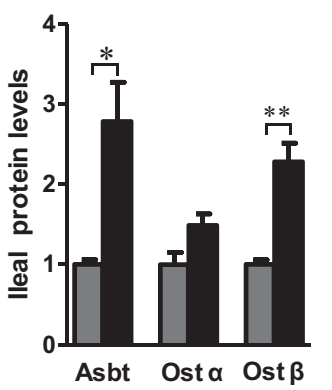

G



E

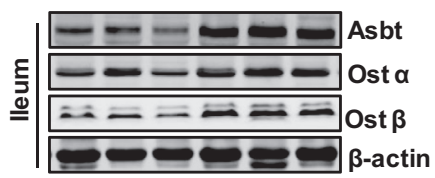

Figure 4 Pharmacologic activation of CAR alters relative mRNA levels of genes involved in bile acid transport. A: Real-time PCR analysis of hepatic expression of genes involved in bile acids transport. B: Urinary level of bile acids. C: Serum level of bile acids. D: Ileal expression of bile acid transporters. E: Protein expression of ileal bile acid transporters was measured by Western blot analysis. F: Quantitative determination of band intensity in $\mathbf{E}$. G: Fecal output of bile acids. Data are expressed as means \pm SEM. $n=5$ to 6 for each group. ${ }^{*} P<0.05,{ }^{*} P<<0.01$. CAR, constitutive androstane receptor; $\mathrm{CD}$, chow diet; LGD, lithogenic diet; TC, TCPOBOP or 1,4-bis[2-(3,5-dichlorpyridyloxy)] benzene; VEH, vehicle.
Asbt Ost $\alpha$ Ost $\beta$

significantly decreased the mRNA and protein expression of Abcg5 and Abcg8 induced by the LGD (Figure 3, A, F and $\mathrm{G})$, which agreed with reduced cholesterol levels in the gallbladder and hepatic bile flow. When profiling cholesterol-metabolizing genes, we found the expression of cholesterol 7a-hydroxylase (Cyp7a1), a key gene involved in the classic BA synthesis pathway, was higher in the liver of TCPOBOP-treated mice (Figure 3B). LGD feeding suppressed Cyp7a1 expression. However, this suppression of Cyp7a1 expression was markedly reversed by CAR activation. Western blot analysis also demonstrated a significant increase in Cyp7al protein expression in the liver of TCPOBOP-treated mice (Figure 3, F and G). The expression of other BA synthetic genes such as Cyp8b1, Cyp7b1, and Cyp27a1 were not changed. This finding agreed with a previous report that overexpression of Cyp7a1 prevented CGD. ${ }^{22}$

To understand the mechanism of reversed Cyp7a1 expression with TCPOBOP treatment, we measured the expression of Shp and ileal Fgf15, two farnesoid X receptor (FXR) target genes that play an important role in negative feedback regulation of Cyp7a1 expression. ${ }^{6-8}$ In the liver, Shp expression was unchanged (Supplemental Figure S3), suggesting the increased Cyp7a1 expression was not caused by hepatic expression of Shp. The hepatic BA levels were not changed either (Supplemental Figure S4A). In the ileum, the mRNA levels of Shp and Fgf15 were significantly decreased after TCPOBOP treatment (Figure 3C). Fgf15 is secreted from the intestine into the blood circulation, then binds to the hepatic Fgfr4, and suppresses Cyp7a1 expression. ${ }^{7}$ Activation of CAR had little effect on Fgfr4 expression with the basal and LGD conditions (Figure 3D). However, the expression of $\beta$-klotho, a coreceptor of Fgfr4, was significantly inhibited in TCPOBOPtreated mice with an LGD (Figure 3D). Consistent with the mRNA level, the protein expression of Fgf15 was also decreased as illustrated by Western blot analysis (Figure 3, $\mathrm{F}$ and $\mathrm{H}$ ). The decreased Fgf15 and $\beta$-klotho expression 
may coordinately relieve the suppression of this signal on Cyp7a1 transcription. Consistent with higher conversion from cholesterol into BAs, lipid analysis revealed that the hepatic cholesterol level was significantly decreased (Figure 3E).

\section{Activation of CAR Induces the Systematic Expression of Genes Involved in BA Transport}

BAs are controlled by an elaborate network of BA transporters. In the biliary secretion system, TCPOBOP treatment had no effect on the expression of Bsep, regardless of diet, but significantly induced Mrp2 expression with a CD (Figure 4A). For sinusoid export transporters, TCPOBOP induced Mrp3 and Mrp4 mRNA levels 2.4- and 23.9-fold with a CD, respectively. When challenged with an LGD, Mrp3 and Mrp4 levels were increased 1.7- and 13.4-fold, respectively, in TCPOBOP-treated mice. This finding is consistent with the notion that Mrp4 was a target gene of CAR. ${ }^{23}$ With a CD, TCPOBOP significantly up-regulated the expression of Oatp2 and sodium $\left(\mathrm{Na}^{+}\right)$-taurocholate cotransport protein, two transporters for hepatic uptake of BAs. However, activation of CAR had no effect on these two genes after an LGD. TCPOBOP treatment robustly inhibited the expression of Oatp1 regardless of the diet. Consistent with the induction of Mrp3/4, the urinary BA outputs (Figure 4B) were drastically increased in LGD-fed TCPOBOP-treated mice; however, the serum BA levels were unchanged (Figure 4C).

More than $90 \%$ of BA is reabsorbed in the ileum; therefore, we also profiled the expression of intestinal genes involved in BA transport. The LGD significantly suppressed the expression of Asbt regardless of drug treatment (Figure 4D). TCPOBOP treatment did not affect Asbt mRNA expression compared with vehicle treatment. TCPOBOP increased Ost $\beta$ mRNA expression with a CD but not with a LGD. The LGD markedly decreased the Mrp3 mRNA level in TCPOBOP-treated mice. The mRNA expression of Ost $\alpha$ was unchanged among the four groups of mice. Although no changes were observed in the mRNA expression of these ileal BA transporters, Western blot analysis revealed that TCPOBOP dramatically increased Asbt and Ost $\beta$ protein expression in LGD-fed mice (Figure 4, E and F). It indicated that activation of CAR increased $\mathrm{BA}$ reabsorption in the ileum of the mice consuming a LGD. Consistent with these facts, we also found the average fecal BA excretion was significantly reduced in TCPOBOP-treated mice at day 9 of the LGD (Figure 4G).

\section{Activation of CAR Decreases the Expression of Genes Involved in Phospholipid Transport}

Although activation of CAR protected mice against CGD, we observed decreased phospholipid secretion into the gallbladder (Figure 2G). The level of Abcb4, a major

\section{$\square$ CD-VEH $\square$ LGD-VEH $\square$ CD-TC $\square$ LGD-TC}

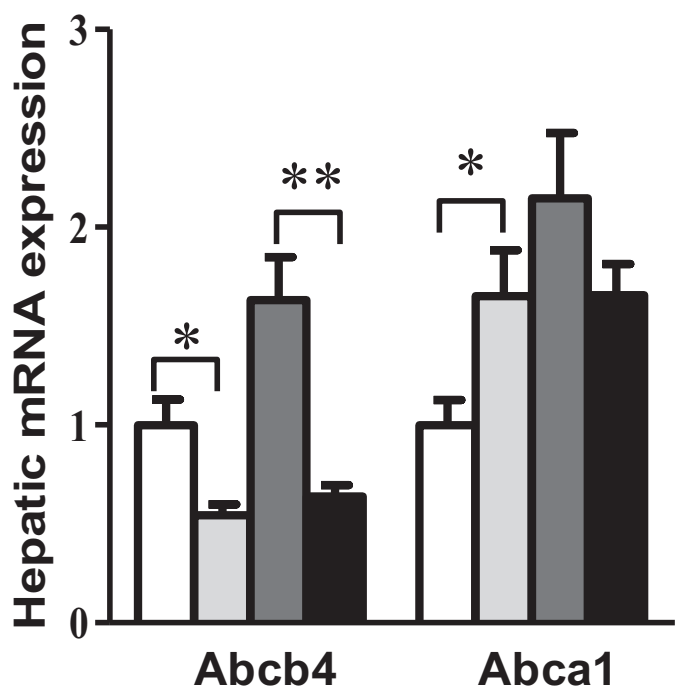

Figure 5 Activation of CAR decreases the expression of phospholipid transporters. Real-time PCR analysis of hepatic mRNA expression of phospholipid transporters. Data are expressed as means \pm SEM. $n=5$ to 6 for each group ${ }^{*} P<0.05,{ }^{*} P P<0.01$. CAR, constitutive androstane receptor; CD, chow diet; LGD, lithogenic diet; TC, TCPOBOP or 1,4-bis-[2-(3,5dichlorpyridyloxy)] benzene; VEH, vehicle.

phospholipid transporter, was markedly decreased with both a CD and LGD in TCPOBOP-treated animals (Figure 5). The level of Abcal was slightly increased with a $\mathrm{CD}$ but unchanged with an LGD.

\section{Activation of CAR Decreases the Expression of Genes Involved in Lipogenesis}

CAR is an antiobesity nuclear receptor. ${ }^{24}$ In our study, TCPOBOP treatment significantly decreased the expression of a gene involved in lipogenesis, sterol regulatory element-binding protein (Srebp-1c), regardless of diet. The expression of stearoyl-CoA desaturase-1 (Scd-1) was unregulated with a $C D$ but markedly suppressed with an LGD (Figure 6A). TCPOBOP did not change hepatic tissue architecture in mice maintained on a CD (Figure 6B). With an LGD, the livers of vehicle-treated mice were filled with large lipid droplets by evidence of both macrovesicular and microvesicular steatosis. However, TCPOBOP-treated mice showed less lipid accumulation, which was further illustrated by Oil Red $\mathrm{O}$ staining of the liver (Figure 6C). Biochemical analysis revealed that, as expected, LGD feeding increased hepatic triglyceride levels, and TCPOBOP treatment decreased hepatic triglyceride content both with a CD and LGD compared with vehicle-treated mice (Figure 6D). Similarly, serum triglycerides were decreased in TCPOBOP-treated mice, whereas the circulating cholesterol was slightly increased (Supplemental Figure S4B). 
A
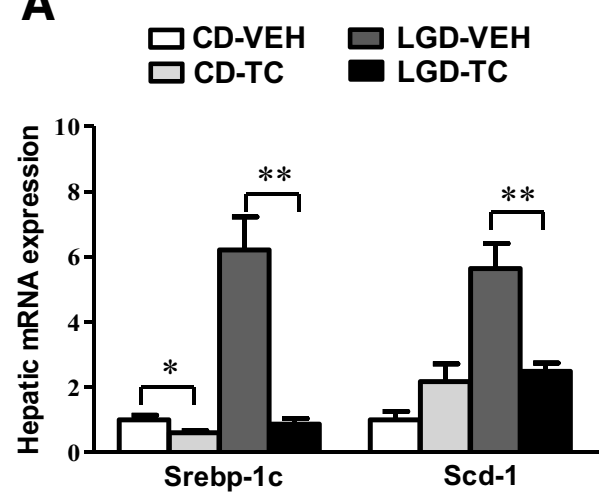

C

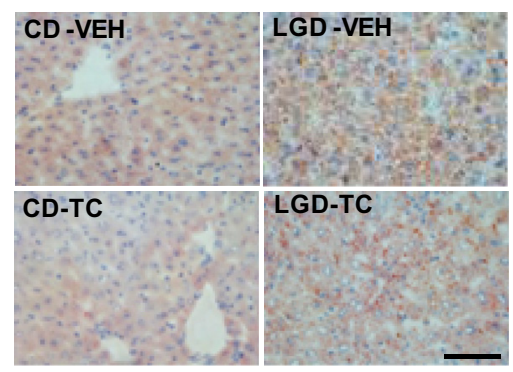

B

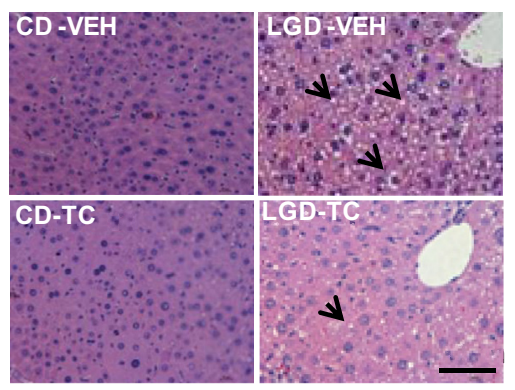

D

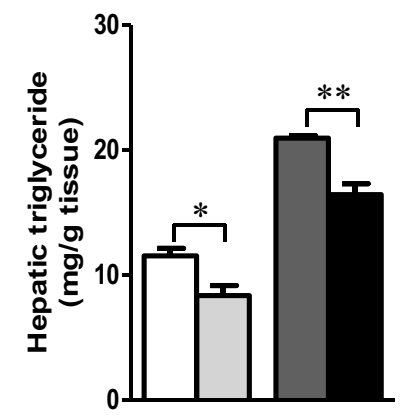

Figure 6 Pharmacologic activation of CAR decreases hepatic steatosis. A: Real-time PCR analysis of hepatic mRNA expression of genes involved in lipogenesis. B: Representative images of H\&E-stained sections of livers. Arrows indicate macrovesicular and microvesicular steatosis. C: $0 i l$ Red 0 staining of liver sections. D: Analysis of hepatic triglycerides level. Data are expressed as means \pm SEM. $n=5$ to 6 for each group (A and D). ${ }^{*} P<0.05,{ }^{*} P P<0.01$. Scale bars $=50 \mu \mathrm{m}$ (B and C). CAR, constitutive androstane receptor; $C D$, chow diet; H\&E, hematoxylin and eosin; LGD, lithogenic diet; TC, ТСРОВOP or 1,4-bis-[2-(3,5dichlorpyridyloxy)] benzene; VEH, vehicle.

\section{Activation of CAR Prevents the LXR $\alpha$-Sensitized Gallstone Disease}

It was reported that activation of $\mathrm{LXR} \alpha$ sensitized mice to gallbladder cholesterol crystallization by increasing cholesterol transport to the gallbladder. ${ }^{25}$ We were interested in whether CAR activation can attenuate gallbladder cholesterol crystallization caused by $\operatorname{LXR} \alpha$ agonist in vivo. Activation of $L X R \alpha$ sensitized mice to gallstone disease, similar to a previous report. ${ }^{25}$ However, TCPOBOP treatment attenuated the aggravated gallbladder cholesterol crystallization induced by GW3965 (Figure 7, A and B). The cholesterol transporters Abcg5 and Abcg8 were highly induced by LXR $\alpha$ (Figure 7C). However, this induction was significantly inhibited by CAR activation (Figure 7C).

\section{Discussion}

Previous studies have shown that CAR played important roles in reverse cholesterol transport, atherosclerosis, and BA elimination. ${ }^{13,15,26}$ However, the role of CAR in CGD is still unknown. In this study, we showed that activation of CAR prevented cholesterol gallstone formation in mice consuming an LGD. The protective role was associated with the markedly decreased secretion of cholesterol into the gallbladder. Although the CSI of bile in the gallbladder did not change, the prevalence of cholesterol gallstones was markedly decreased in TCPOBOP-treated mice. In the liver, the relative greatly decreased biliary cholesterol level and slightly decreased phospholipid secretion, combined with unchanged BA secretion, contributed to the decreased CSI of hepatic fresh bile, thus being antilithogenic. Activation of CAR also protected mice against $\mathrm{LXR} \alpha$-sensitized gallstone formation. Although we showed that the activation of CAR by TCPOBOP had beneficial effects with the CGD, this study is limited because CAR knockout mice were not used to evaluate whether TCPOBOP effect is CAR dependent. In addition, CAR knockout mice should be used to determine whether ablation of this receptor sensitized mice to gallstone disease in the future.

Recently, the role of CAR in cholesterol transport was highlighted, especially the role in reverse cholesterol transport and atherosclerosis. Therefore, CAR may play an important role in whole-body cholesterol homeostasis. Indeed, activation of CAR can decrease high-density lipoprotein cholesterol level, the main component in the reverse transport of cholesterol to the liver. ${ }^{27}$ However, CAR activation can also stimulate reverse cholesterol transport and can protect mice against atherosclerosis along with decreased biliary cholesterol level by repressing the expression of the Abcg5/8 cholesterol transporters. ${ }^{13}$ In agreement, we found decreased biliary secretion of cholesterol into the gallbladder of TCPOBOP-treated mice as well as the significantly suppressed mRNA and protein levels of Abcg5/8, which are required for essential secretion of cholesterol into bile. In addition, we observed a slight reduction in phospholipid secretion in bile of TCPOBOP-treated mice. This finding 
could be explained by the decreased biliary export of cholesterol. Abcg5/8 required Abcb4 for efficient secretion of cholesterol from liver into bile. ${ }^{28} \mathrm{We}$ also found that altered hepatic secretion of biliary lipids is a prerequisite for cholesterol supersaturated bile in the gallbladder and

A
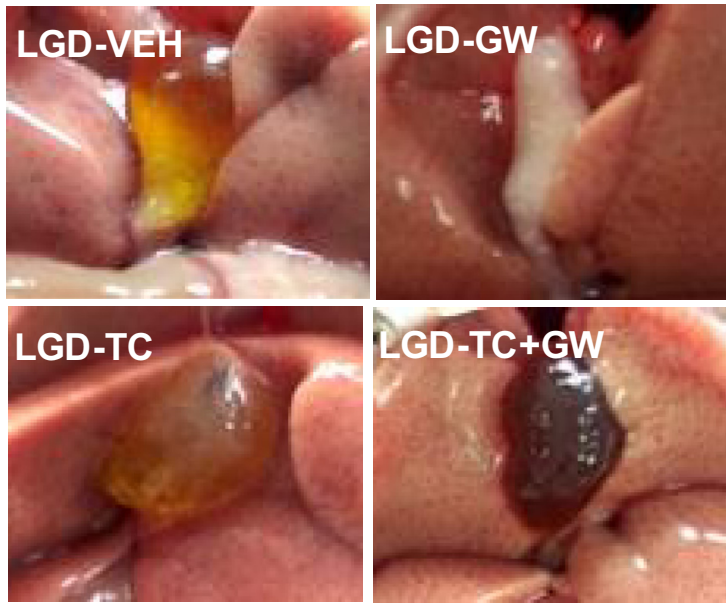

B
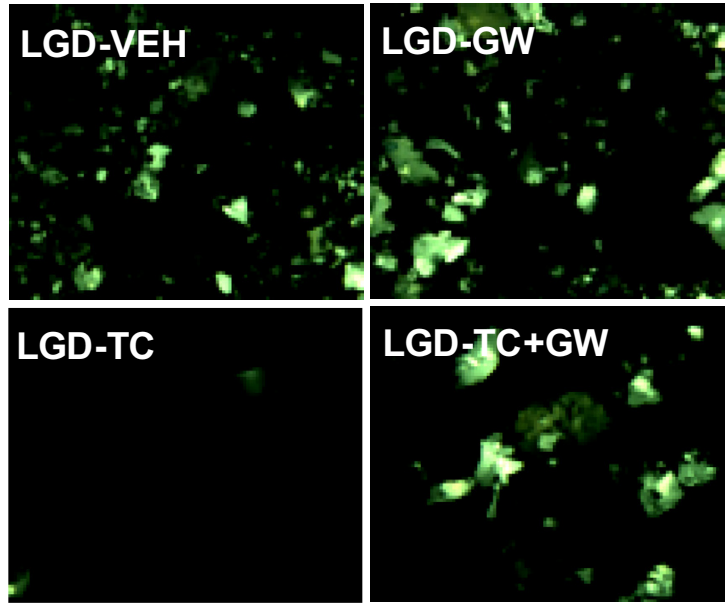

C
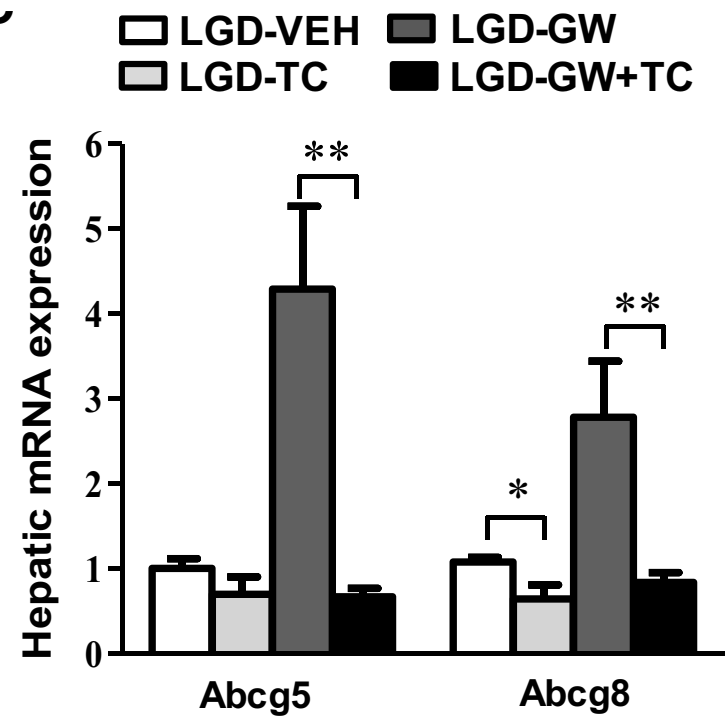

subsequent cholesterol gallstone formation. ${ }^{29}$ Therefore, the decreased CSI of the hepatic bile in TCPOBOP-treated mice precedes the effect of CAR on protecting cholesterol gallstone formation, for a pathophysiological explanation of the protective role of CAR activation in the lithogenic phenotype.

We found that an LGD markedly inhibited the expression of Cyp7a1 (the rate-limiting enzyme of BA biosynthesis), which was consistent with a previous study. ${ }^{25}$ This finding is due to the presence of BAs in the LGD, which may signal the negative feedback mechanism of regulating BAs. ${ }^{30}$ The expression of Cyp7a1 was significantly higher in TCPOBOP-treated mice than in vehicle-treated mice regardless of diet. Miao et $\mathrm{al}^{31}$ reported that activation of CAR inhibited Cyp7a1 expression, which is opposite to our results. We reasoned this discrepancy may be the result of different mouse strains used. BALB/c mice were used in their study, whereas we used C57BL/6J mice. It is also possible that a different dosage of TCPOBOP might have a different effect on Cyp7a1 expression (TCPOBOP $0.3 \mathrm{mg} / \mathrm{kg}$ body weight in their study and $1.0 \mathrm{mg} / \mathrm{kg}$ body weight in ours). Cyp7a1 is negatively regulated by Shp signaling in the liver $^{32}$ and Fgf15 from the intestine. ${ }^{7}$ We found the hepatic expression of Shp did not changed, which agreed with the constant hepatic BA level (Supplemental Figure S4A). However, the ileal expression of Shp and Fgf15 was significantly suppressed by the TCPOBOP treatment. The lower level of Fgf15 could weaken negative feedback and thereby resulted in higher expression of Cyp7a1.

Previous studies have shown that CAR played an important role in xenobiotic molecular elimination and also controlled the metabolism of endogenous compounds such as BAs and triglycerides. ${ }^{26,33,34}$ Despite the activation of Cyp7a1, our TCPOBOP-treated mice showed a constant biliary level of BAs, which was due to the unchanged Bsep and Mrp2 expression that mediated canalicular BA efflux. With increased synthesis of BAs in the liver, CAR activation decreased hepatic BA uptake. TCPOBOP treatment robustly suppressed the expression of Oatp1, thereby indicating a decreased hepatic $\mathrm{Na}^{+}$-independent import of BAs. The increased BA synthesis but constant biliary BA level in TCPOBOP-treated mice might be due to the overwhelming up-regulation of Mrp4 (ABCC4), a target gene of CAR, which is responsible for the sinusoidal efflux of BAs from the hepatocytes into the space of Disse. ${ }^{23,35}$ When mice were maintained on a western-type diet, Sberna et $\mathrm{al}^{13}$ found

Figure 7 Activation of CAR prevents the LXR $\alpha$-sensitized gallstone disease. A: Gross appearance of representative gallbladders in $\mathrm{C57 \textrm {BL } / 6 \mathrm { J }}$ mice treated with VEH, TC $(1.0 \mathrm{mg} / \mathrm{kg}$ body weight, once per week), and/or GW (30 mg/kg body weight, once per day). All mice were fed a LGD for 2 weeks. B: Polarizing light microscopy of cholesterol crystals. C: Real-time PCR analysis of hepatic mRNA expression of cholesterol transporters Abcg 5 and Abcg8. Data are expressed as means \pm SEM. $n=5$ for each group. ${ }^{*} P<0.05,{ }^{* *} P<0.01$. Original magnification, $\times 100$ (B). CAR, constitutive androstane receptor; CD, chow diet; GW, GW3965; LGD, lithogenic diet; LXR $\alpha$, liver $X$ receptor $\alpha$; TC, TCPOBOP or 1,4-bis-[2-(3,5dichlorpyridyloxy)] benzene; VEH, vehicle. 
that activation of CAR by TCPOBOP stimulated fecal BA excretion. In our results, TCPOBOP treatment suppressed the fecal excretion of BAs. In their study, CAR activation increased the sinusoidal efflux and enhanced the hepatic BA uptake. In addition, the canalicular membrane secretion of BAs was significantly increased, which is essential to the stimulated fecal excretion of BAs. In our study, we found that the protein levels of ileal BA transporters Asbt and Ost $\beta$ were significantly higher in TCPOBOP-treated mice (Figure 4, E and F). The higher expression of Asbt and Ost $\beta$ will increase the reabsorption of $\mathrm{BA}$ and subsequently decrease fecal BA excretion. We reasoned this discrepancy might be due to the presence of cholate in the diet. The cholate will signal a negative feedback on Asbt, Ost $\alpha$, and Ost $\beta$ expression. However, this negative feedback might be relieved when CAR is activated.

Several nuclear receptors affect lithogenesis and include FXR, LXR, pregnane X receptor (PXR), and estrogen receptors. ${ }^{10,25,36-38}$ We found that CAR played an antilithogenic role in lithogenesis similar to FXR and PXR. However, the mechanisms were quite different. CAR activation played a central role in canalicular membrane transport of cholesterol, whereas FXR activation had profound effects on canalicular BA and phospholipid secretion. ${ }^{36}$ In addition, TCPOBOP induced the conversion from cholesterol into BAs, whereas FXR suppressed the expression of Cyp7a1.

Here, we demonstrated that activation of CAR by TCPOBOP prevents cholesterol gallstone formation via inhibiting cholesterol transport from the liver to the gallbladder. PXR, a sister xenobiotic receptor of CAR, also prevents CGD. ${ }^{10}$ Like in the case of drug metabolism, the function of these two xenobiotic receptors in CGD is also conserved. CAR and PXR share many of their target genes and indirectly regulated genes. Activation of PXR prevented CGD by decreasing biliary concentrations of cholesterol and increasing the level of bile salts. In contrast to PXR, activation of CAR prevented CGD by decreasing biliary concentrations of cholesterol without changing the level of bile salts. Mechanistically, both receptors decreased cholesterol secretion through inhibiting the expression of Abcg5/8. In contrast to the unchanged biliary BA level in CAR-activated mice, PXR activation increased biliary BAs via induction of Mrp2 without Bsep. In the intestine, both PXR and CAR increased BAs reabsorption by increasing Asbt expression. Thus, both receptors provide a rationale for pharmacologic interference with CGD via these nuclear receptors. The present and previous studies establish a crucial and collective role for PXR and CAR in preventing CGD in vivo. ${ }^{10}$ We conclude that CAR and PXR mediate overlapping yet distinct defense to prevent CGD. Combined loss of PXR and CAR heightens the sensitivity to toxic BAs in mice. ${ }^{39}$ It is also tempting to speculate that a combined defect in PXR and CAR expression and/or activation may increase and/or decrease susceptibility to CGD and may present a novel research topic.
LXR $\alpha$ has a lithogenic effect opposite to that of CAR. The lithogenic effect of $\operatorname{LXR} \alpha$ was due to the combined effect of increased biliary cholesterol and phospholipids and decreased biliary BAs. ${ }^{25}$ In our study, we found markedly decreased hepatic secretion of cholesterol, slightly decreased phospholipid secretion, and unchanged BA secretion. Activation of $\mathrm{LXR} \alpha$ suppresses $\mathrm{CAR}$-mediated xenobiotic response, and, conversely, activation of CAR inhibits the LXR $\alpha$-mediated lipogenesis. ${ }^{40}$ Indeed, we observed that the hepatic triglyceride level was markedly decreased after a 2-week TCPOBOP treatment, probably because of the suppressed expression of Srebp-1c and Scd-1 in the liver. Activation of CAR also protected the mice from LXR $\alpha$-sensitized gallstone disease by suppressing the expression of Abcg5 and Abcg8 induced by LXR $\alpha$. Our findings expand the cognitive territory of the checks and balances behind $\operatorname{LXR} \alpha$ and CAR.

\section{Conclusions}

We provide evidence that CAR may be a new factor in preventing cholesterol gallstone formation in a mouse model under lithogenic conditions. Activation of CAR may represent a new approach for treating CGD. Certainly, whether CAR could be a novel target in preventing or treating CGD in humans remains to be further determined.

\section{Acknowledgments}

S.C., M.Z., and J.H. designed and performed experiments and wrote the manuscript; Q.L., J.K., J.S., S.P., L.C., H.L., T.W., and R.L. helped with experiments; Y.L., W.J., and Z.Z. contributed to the Discussion and reviewed the manuscript; J.H. obtained funding, designed experiments, and wrote the manuscript; S.C., M.Z., and J.H. are the guarantors of this work and, as such, had full access to all of the data in the study and take responsibility for the integrity of the data and the accuracy of the data analysis.

\section{Supplemental Data}

Supplemental material for this article can be found at http://dx.doi.org/10.1016/j.ajpath.2016.12.013.

\section{References}

1. Claudel T, Zollner G, Wagner M, Trauner M: Role of nuclear receptors for bile acid metabolism, bile secretion, cholestasis, and gallstone disease. Biochim Biophys Acta 2011, 1812:867-878

2. Graf GA, Yu L, Li WP, Gerard R, Tuma PL, Cohen JC, Hobbs HH: ABCG5 and ABCG8 are obligate heterodimers for protein trafficking and biliary cholesterol excretion. J Biol Chem 2003, 278:48275-48282

3. Yu L, Gupta S, Xu F, Liverman AD, Moschetta A, Mangelsdorf DJ, Repa JJ, Hobbs HH, Cohen JC: Expression of ABCG5 and ABCG8 is required for regulation of biliary cholesterol secretion. J Biol Chem 2005, 280:8742-8747 
4. Smit JJ, Schinkel AH, Oude Elferink RP, Groen AK, Wagenaar E, van Deemter L, Mol CA, Ottenhoff R, van der Lugt NM, van Roon MA, van der Valk MA, Offerhaus GJ, Berns AJ, Borst P: Homozygous disruption of the murine mdr2 P-glycoprotein gene leads to a complete absence of phospholipid from bile and to liver disease. Cell 1993, 75:451-462

5. de Aguiar Vallim TQ, Tarling EJ, Ahn H, Hagey LR, Romanoski CE, Lee RG, Graham MJ, Motohashi H, Yamamoto M, Edwards PA: MAFG is a transcriptional repressor of bile acid synthesis and metabolism. Cell Metab 2015, 21:298-310

6. Kerr TA, Saeki S, Schneider M, Schaefer K, Berdy S, Redder T, Shan B, Russell DW, Schwarz M: Loss of nuclear receptor SHP impairs but does not eliminate negative feedback regulation of bile acid synthesis. Dev Cell 2002, 2:713-720

7. Inagaki $\mathrm{T}$, Choi M, Moschetta A, Peng L, Cummins CL, McDonald JG, Luo G, Jones SA, Goodwin B, Richardson JA, Gerard RD, Repa JJ, Mangelsdorf DJ, Kliewer SA: Fibroblast growth factor 15 functions as an enterohepatic signal to regulate bile acid homeostasis. Cell Metab 2005, 2:217-225

8. Inagaki T, Moschetta A, Lee YK, Peng L, Zhao G, Downes M, Yu RT, Shelton JM, Richardson JA, Repa JJ, Mangelsdorf DJ, Kliewer SA: Regulation of antibacterial defense in the small intestine by the nuclear bile acid receptor. Proc Natl Acad Sci U S A 2006, 103:3920-3925

9. Dawson PA, Lan T, Rao A: Bile acid transporters. J Lipid Res 2009, 50:2340-2357

10. He J, Nishida S, Xu M, Makishima M, Xie W: PXR prevents cholesterol gallstone disease by regulating biosynthesis and transport of bile salts. Gastroenterology 2011, 140:2095-2106

11. Wei P, Zhang J, Egan-Hafley M, Liang S, Moore DD: The nuclear receptor CAR mediates specific xenobiotic induction of drug metabolism. Nature 2000, 407:920-923

12. Qatanani M, Moore DD: CAR, the continuously advancing receptor, in drug metabolism and disease. Curr Drug Metab 2005, 6:329-339

13. Sberna AL, Assem M, Gautier T, Grober J, Guiu B, Jeannin A, Pais de Barros JP, Athias A, Lagrost L, Masson D: Constitutive androstane receptor activation stimulates faecal bile acid excretion and reverse cholesterol transport in mice. J Hepatol 2011, 55:154-161

14. Lickteig AJ, Csanaky IL, Pratt-Hyatt M, Klaassen CD: Activation of constitutive androstane receptor (CAR) in mice results in maintained biliary excretion of bile acids despite a marked decrease of bile acids in liver. Toxicol Sci 2016, 151:403-418

15. Sberna AL, Assem M, Xiao R, Ayers S, Gautier T, Guiu B, Deckert V, Chevriaux A, Grober J, Le Guern N, Pais de Barros JP, Moore DD, Lagrost L, Masson D: Constitutive androstane receptor activation decreases plasma apolipoprotein B-containing lipoproteins and atherosclerosis in low-density lipoprotein receptor-deficient mice. Arterioscler Thromb Vasc Biol 2011, 31:2232-2239

16. Biddinger SB, Haas JT, Yu BB, Bezy O, Jing E, Zhang W, Unterman TG, Carey MC, Kahn CR: Hepatic insulin resistance directly promotes formation of cholesterol gallstones. Nat Med 2008, 14:778-782

17. Carey MC: Critical tables for calculating the cholesterol saturation of native bile. J Lipid Res 1978, 19:945-955

18. Yu C, Wang F, Kan M, Jin C, Jones RB, Weinstein M, Deng CX, McKeehan WL: Elevated cholesterol metabolism and bile acid synthesis in mice lacking membrane tyrosine kinase receptor FGFR4. J Biol Chem 2000, 275:15482-15489

19. Folch J, Lees M, Sloane Stanley GH: A simple method for the isolation and purification of total lipides from animal tissues. J Biol Chem 1957, 226:497-509

20. Ma K, Xiao R, Tseng HT, Shan L, Fu L, Moore DD: Circadian dysregulation disrupts bile acid homeostasis. PLoS One 2009, 4:e6843

21. Russell DW: The enzymes, regulation, and genetics of bile acid synthesis. Annu Rev Biochem 2003, 72:137-174

22. Miyake JH, Duong-Polk XT, Taylor JM, Du EZ, Castellani LW, Lusis AJ, Davis RA: Transgenic expression of cholesterol-7-alphahydroxylase prevents atherosclerosis in $\mathrm{C} 57 \mathrm{BL} / 6 \mathrm{~J}$ mice. Arterioscler Thromb Vasc Biol 2002, 22:121-126
23. Assem M, Schuetz EG, Leggas M, Sun D, Yasuda K, Reid G, Zelcer N, Adachi M, Strom S, Evans RM, Moore DD, Borst P, Schuetz JD: Interactions between hepatic Mrp4 and Sult2a as revealed by the constitutive androstane receptor and Mrp4 knockout mice. J Biol Chem 2004, 279:22250-22257

24. Gao J, He J, Zhai Y, Wada T, Xie W: The constitutive androstane receptor is an anti-obesity nuclear receptor that improves insulin sensitivity. J Biol Chem 2009, 284:25984-25992

25. Uppal H, Zhai Y, Gangopadhyay A, Khadem S, Ren S, Moser JA, Xie W: Activation of liver $\mathrm{X}$ receptor sensitizes mice to gallbladder cholesterol crystallization. Hepatology 2008, 47:1331-1342

26. Wagner M, Halilbasic E, Marschall HU, Zollner G, Fickert P, Langner C, Zatloukal K, Denk H, Trauner M: CAR and PXR agonists stimulate hepatic bile acid and bilirubin detoxification and elimination pathways in mice. Hepatology 2005, 42:420-430

27. Masson D, Qatanani M, Sberna AL, Xiao R, Pais de Barros JP, Grober J, Deckert V, Athias A, Gambert P, Lagrost L, Moore DD, Assem M: Activation of the constitutive androstane receptor decreases HDL in wild-type and human apoA-I transgenic mice. J Lipid Res 2008, 49:1682-1691

28. Langheim S, Yu L, von Bergmann K, Lutjohann D, Xu F, Hobbs HH, Cohen JC: ABCG5 and ABCG8 require MDR2 for secretion of cholesterol into bile. J Lipid Res 2005, 46:1732-1738

29. Wang DQ, Lammert F, Paigen B, Carey MC: Phenotypic characterization of lith genes that determine susceptibility to cholesterol cholelithiasis in inbred mice. Pathophysiology of biliary lipid secretion. J Lipid Res 1999, 40:2066-2079

30. Kim I, Ahn SH, Inagaki T, Choi M, Ito S, Guo GL, Kliewer SA, Gonzalez FJ: Differential regulation of bile acid homeostasis by the farnesoid X receptor in liver and intestine. J Lipid Res 2007, 48: $2664-2672$

31. Miao J, Fang S, Bae Y, Kemper JK: Functional inhibitory cross-talk between constitutive androstane receptor and hepatic nuclear factor-4 in hepatic lipid/glucose metabolism is mediated by competition for binding to the DR1 motif and to the common coactivators, GRIP-1 and PGC-1alpha. J Biol Chem 2006, 281:14537-14546

32. Kir S, Zhang Y, Gerard RD, Kliewer SA, Mangelsdorf DJ: Nuclear receptors HNF4alpha and LRH-1 cooperate in regulating Cyp7al in vivo. J Biol Chem 2012, 287:41334-41341

33. Guo GL, Lambert G, Negishi M, Ward JM, Brewer HB Jr, Kliewer SA, Gonzalez FJ, Sinal CJ: Complementary roles of farnesoid $\mathrm{X}$ receptor, pregnane $\mathrm{X}$ receptor, and constitutive androstane receptor in protection against bile acid toxicity. J Biol Chem 2003, 278: 45062-45071

34. Maglich JM, Lobe DC, Moore JT: The nuclear receptor CAR (NR1I3) regulates serum triglyceride levels under conditions of metabolic stress. J Lipid Res 2009, 50:439-445

35. Mennone A, Soroka CJ, Cai SY, Harry K, Adachi M, Hagey L, Schuetz JD, Boyer JL: Mrp4-/- mice have an impaired cytoprotective response in obstructive cholestasis. Hepatology 2006, 43:10131021

36. Moschetta A, Bookout AL, Mangelsdorf DJ: Prevention of cholesterol gallstone disease by FXR agonists in a mouse model. Nat Med 2004, 10:1352-1358

37. Wang HH, Afdhal NH, Wang DQ: Estrogen receptor alpha, but not beta, plays a major role in 17beta-estradiol-induced murine cholesterol gallstones. Gastroenterology 2004, 127:239-249

38. de Bari O, Wang TY, Liu M, Portincasa P, Wang DQ: Estrogen induces two distinct cholesterol crystallization pathways by activating ERalpha and GPR30 in female mice. J Lipid Res 2015, 56:1691-1700

39. Uppal H, Toma D, Saini SP, Ren S, Jones TJ, Xie W: Combined loss of orphan receptors PXR and CAR heightens sensitivity to toxic bile acids in mice. Hepatology 2005, 41:168-176

40. Xiao L, Xie X, Zhai Y: Functional crosstalk of CAR-LXR and RORLXR in drug metabolism and lipid metabolism. Adv Drug Deliv Rev 2010, 62:1316-1321 